PAIN

\title{
Is alcohol consumption related to likelihood of reporting chronic widespread pain in people with stable consumption? Results from UK Biobank

\author{
--Manuscript Draft--
}

\begin{tabular}{|c|c|}
\hline Manuscript Number: & PAIN-D-14-14682R2 \\
\hline Article Type: & Research Paper \\
\hline Section/Category: & Epidemiology \\
\hline Keywords: & alcohol; chronic pain; epidemiology \\
\hline Corresponding Author: & $\begin{array}{l}\text { Marcus Beasley, MSc } \\
\text { University of Aberdeen Institute of Applied Health Sciences } \\
\text { Aberdeen, Please Select UNITED KINGDOM }\end{array}$ \\
\hline First Author: & Marcus Beasley, MSc \\
\hline \multirow[t]{3}{*}{ Order of Authors: } & Marcus Beasley, MSc \\
\hline & Tatiana Macfarlane, $\mathrm{PhD}$ \\
\hline & Gary Macfarlane, BSc(Hons), MBChB, PhD, CStat, MD(Hons), FFPHM \\
\hline Abstract: & $\begin{array}{l}\text { Studies have suggested alcohol consumption is strongly related to reduced reporting of } \\
\text { chronic widespread pain (CWP) and level of disability in people with CWP or } \\
\text { fibromyalgia. Direction of causality has not been established, that is whether the } \\
\text { association is due to people's health influencing their alcohol consumption or vice } \\
\text { versa. UK Biobank recruited over } 500,000 \text { people aged } 40-69 \text { years registered at } \\
\text { medical practices nationwide. Participants provided detailed information on health and } \\
\text { lifestyle factors including pain and alcohol consumption. Total units consumed per } \\
\text { week was calculated for current drinkers. Information was also collected on changes in } \\
\text { alcohol consumption and reasons for such changes. Analysis was by logistic } \\
\text { regression expressed as odds ratios (ORs) with } 95 \% \text { confidence intervals (Cls), then } \\
\text { adjusted for a large number of potential confounding factors (adjORs). In males who } \\
\text { reported drinking the same as } 10 \text { years previously, there was a U-shaped relationship } \\
\text { between amount drunk and odds of reporting CWP (non-drinkers CWP prevalence } \\
2.4 \%, 19.1-32.1 \text { units/wk } 0.4 \%,>53.6 \text { units/wk } 1.0 \% \text {; adjORs } 2.5395 \% \text { Cl [1.78-3.60] } \\
\text { vs } 1 \text { vs } 1.52 \text { [1.05-2.20]). In females there was a decrease in proportion reporting CWP } \\
\text { up to the modal category of alcohol consumption with no further change in those } \\
\text { drinking more (non-drinkers CWP prevalence } 3.4 \%, 6.4-11.2 \text { units/wk } 0.7 \%,>32.1 \\
\text { units/wk } 0.7 \% \text {; adjORs } 2.11 \text { [1.67-2.66] vs } 1 \text { vs } 0.86 \text { [0.54-1.39]). This large study has } \\
\text { shown a clear relationship between alcohol consumption and reporting of pain even in } \\
\text { people who had not reported changing consumption due to health concerns, after } \\
\text { adjustment for potential confounding factors. }\end{array}$ \\
\hline
\end{tabular}


Marcus Beasley

The Institute of Applied Health Sciences

University of Aberdeen

Polwarth Building, Aberdeen

Scotland, United Kingdom

AB25 2ZD

Dear editors of PAIN,

We would like to resubmit the manuscript entitled 'Is alcohol consumption related to likelihood of reporting chronic widespread pain in people with stable consumption? Results from UK Biobank', authored by myself (MB), Tatiana Macfarlane (TM), and Gary Macfarlane (GM). All authors have read and approved the paper. We have revised the manuscript and the version submitted has tracked changes.

Author contributions were as follows:

Study conception and design: MB, TM, GM

Analysis and interpretation of data: MB, TM, GM

Drafting of manuscript: MB

Critical revision: $\mathrm{MB}, \mathrm{TM}, \mathrm{GM}$

Please find below our responses to reviewers' comments on our previous version of the manuscript (reviewers' comments in bold and our replies are in italic).

We believe that attention to these has improved the manuscript and hope that PAIN may accept it for publication.

Sincerely,

Marcus Beasley 
$\underline{\text { Response to Reviewers' Comments }}$

Reviewer \#1: Beasley and colleagues have adequately referred to all my previous points, with one exception. The discussion of the mechanisms by which alcohol mediates pain has simply been moved from the introduction to the discussion. While I agree that it fits much better there, I still think that their explanation does not explain things very well. First, the study by Kim et al. (2013) examines the relationship between alcohol consumption and fibromyalgia and does not examine the mechanisms (i.e. GABA). The reported review by Enna et al. (2006) talks about animals (i.e. relationship between GABA and pain) and the study by Foerster et al. (2012) provides only evidence of altered GABA concentrations in the insula of fibromyalgia patients and does not link those to alcohol consumption. Thus a clear explanation of how GABA reduces pain sensitivity and why insula is still missing.

I would suggest not talking about "mechanisms" but rather more broadly, about "previous studies have shown".

We have removed the paragraph talking about mechanisms.

Reviewer \#2: In my primary review, I commented that the data in this paper speak for themselves: that of course remains the case, but the revision does ease the task of the reader to make sense of them. Overall the authors' responses to the comments of both reviewers have enhanced the paper, in terms of flow of argument and qualifications regarding inferences that can be made.

There remains scope for improving grammar and syntax, which I expect can be rectified in the proofing process.

We've read through the manuscript again to check for grammatical/syntactical errors, and made some changes.

The abstract would be enhanced by explicit expression of the main finding - a clear relationship between the consumption of alcohol and experience of pain even among those who had not reported changing alcohol consumption due to health concerns.

We've rewritten the final sentence of the abstract to try and make the expression of the main finding more explicit as suggested.

Reviewer \#3: Review of revised manuscript PAIN-D-14-14682R1

I have read the manuscript, and comments and proposed changes from reviewers.

The authors have considered comments from reviewers and adjusted the text in, what I consider, a relevant way. In cases where reviewers' suggestions have not been fully adopted the authors have argued for their case in a relevant and valid way (for examples: the comment on multicollinearity, the speculative reasoning about the effect of alcohol on pain).

Comments and suggestions from reviewers have made hypotheses more clear and the discussion more relevant than in the previous version of the manuscript.

Thank you to all the reviewers for the time taken to review this manuscript. Note also, that there has been one minor alteration to the heading for Figure $2 d$ with the addition of the word 'illness'. 
Is alcohol consumption related to likelihood of reporting chronic widespread pain in people with stable consumption? Results from UK Biobank

\section{Abstract}

Studies have suggested alcohol consumption is strongly related to reduced reporting of chronic widespread pain (CWP) and level of disability in people with CWP or fibromyalgia. Direction of causality has not been established, that is whether the association is due to people's health influencing their alcohol consumption or vice versa. UK Biobank recruited over 500,000 people aged 40-69 years registered at medical practices nationwide. Participants provided detailed information on health and lifestyle factors including pain and alcohol consumption. Total units consumed per week was calculated for current drinkers. Information was also collected on changes in alcohol consumption and reasons for such changes. Analysis was by logistic regression expressed as odds ratios (ORs) with $95 \%$ confidence intervals (Cls), then adjusted for a large number of potential confounding factors (adjORs). In males who reported drinking the same as 10 years previously, there was a U-shaped relationship between amount drunk and odds of reporting CWP (non-drinkers CWP prevalence 2.4\%, 19.1-32.1 units/wk 0.4\%, >53.6 units/wk 1.0\%; adjORs $2.5395 \% \mathrm{Cl}$ [1.78-3.60] vs 1 vs 1.52 [1.05-2.20]). In females there was a decrease in proportion reporting CWP up to the modal category of alcohol consumption with no further change in those drinking more (non-drinkers CWP prevalence 3.4\%, 6.411.2 units/wk 0.7\%, >32.1 units/wk 0.7\%; adjORs 2.11 [1.67-2.66] vs 1 vs 0.86 [0.54-1.39]). This large study has shown a clear relationship between alcohol consumption and reporting of pain even in people who had not reported changing consumption due to health concerns, after adjustment for potential confounding factors. 
Is alcohol consumption related to likelihood of reporting chronic widespread pain in people with stable consumption? Results from UK Biobank

Marcus John Beasley ${ }^{1,2 *}$, Tatiana Victorovna Macfarlane ${ }^{1}$, Gary John Macfarlane ${ }^{1,2}$

(1) Epidemiology Group, School of Medicine, Medical Sciences and Nutrition, University of Aberdeen, United Kingdom

(2) Aberdeen Centre for Arthritis and Musculoskeletal Health, University of Aberdeen, United Kingdom

Number of text page of entire manuscript: 23 (including 10 pages containing figures and tables)

Number of figures: 2

Number of tables: 6

*Corresponding Author:

Marcus Beasley

Epidemiology Group, Institute of Applied Health Sciences

School of Medicine and Dentistry, University of Aberdeen

Polwarth Building, Foresterhill

Aberdeen AB25 2ZD

Telephone: $+44(0) 1244437087$

Email: $\underline{\text { m.beasley@abdn.ac.uk }}$

Epidemiology Group: www.abdn.ac.uk/epidemiology

Twitter: @mahkusjaybee 


\section{Abstract}

Studies have suggested alcohol consumption is strongly related to reduced reporting of chronic widespread pain (CWP) and level of disability in people with CWP or fibromyalgia. Direction of causality has not been established, that is whether the association is due to people's health influencing their alcohol consumption or vice versa. UK Biobank recruited over 500,000 people aged 40-69 years registered at medical practices nationwide. Participants provided detailed information on health and lifestyle factors including pain and alcohol consumption. Total units consumed per week was calculated for current drinkers. Information was also collected on changes in alcohol consumption and reasons for such changes. Analysis was by logistic regression expressed as odds ratios (ORs) with $95 \%$ confidence intervals (Cls), then adjusted for a large number of potential confounding factors (adjORs). In males who reported drinking the same as 10 years previously, there was a U-shaped relationship between amount drunk and odds of reporting CWP (non-drinkers CWP prevalence 2.4\%, 19.1-32.1 units/wk 0.4\%, >53.6 units/wk 1.0\%; adjORs $2.5395 \% \mathrm{Cl}$ [1.78-3.60] vs 1 vs 1.52 [1.05-2.20]). In females there was a decrease in proportion reporting CWP up to the modal category of alcohol consumption with no further change in those drinking more (non-drinkers CWP prevalence 3.4\%, 6.411.2 units/wk $0.7 \%,>32.1$ units/wk 0.7\%; adjORs 2.11 [1.67-2.66] vs 1 vs 0.86 [0.54-1.39]). This large study has shown a clearstrong relationship between alcohol consumption and-likelihoof of reporting of pain WWP exists even in people reporting unchanged consumptionwho had not reported changing consumption due to health concerns, and-after adjustment for a large number of -potential confounding factors. 


\section{Introduction}

Chronic widespread pain (CWP) or multi-site pain have been included as essential criteria in classification and diagnostic criteria for fibromyalgia, respectively $[26,27]$. CWP is defined as pain that is present above and below the waist, on the left and right hand sides, and in the axial skeleton. Epidemiological studies have identified a number of characteristics which might be considered risk factors for the development of CWP [9]. These include sleep problems, psychological distress, and certain beliefs about health and illness. Estimates for the prevalence of fibromyalgia range from around $1 \%$ to around $5 \%$ [10], while a recent meta-analysis estimated the pooled prevalence of CWP at $10.6 \%$ [18].

Among the lifestyle factors that have been identified as having an association with CWP is the consumption of alcohol. One population-based UK study has shown pain reporting and pain-related disability is associated with the amount of alcohol usually consumed [15]. In this study of 13,574 people, those who said they had never druank alcohol regularly were $50 \%$ more likely to report CWP than those who said they drank 11-35 units/week. Among people with CWP, those that had never druank regularly were more than twice as likely to have pain that was disabling than those drinking 11-35 units/week. A further US clinic study of patients with fibromyalgia found reduced symptoms and better quality of life in those drinking low to moderate amounts of alcohol than those not drinking [11].

That pain is less common among people who drink alcohol does not mean that the reason they are less likely to have pain is because they drink alcohol. A number of problems with making such causal inferences from observational studies of the relationship between alcohol and health outcomes have been noted ${ }_{-}$including ${ }_{2}$ dependent misclassification of exposure and outcome [2]; unmeasured confounding [12]; the inappropriate selection of referent group [12]; classification biases counting occasional drinkers in abstainer reference group [22]; and, selection biases [12,23]. For the alcoholpain relationship a major issue is whether the observed relationship is due to people reducing or stopping their alcohol consumption because of ill-health [7].

Among criteria for causality that seem to be satisfied by observational evidence of the association between alcohol and pain are strength of relationship, and biological gradient, i.e. dose-response relationship [15]. It has however been noted that the positive effect of alcohol was not restricted to pain reporting but could also be shown with other health outcomes [6] and this lack of specificity would be evidence against a causal effect on pain. One criterion missing from currently reported observational studies is temporality, which would indicate whether drinking precedes relief from 
chronic pain, or alternatively whether experiencing chronic pain leads people to reduce their alcohol consumption.

Using data from a very large "Biobank", which provides information on a comprehensive set of possible confounders and which allows us to identify people with long-term stable alcohol consumption, we wished to test whether we could confirm a previous observation of a 'reversed Jshaped' relationship between alcohol consumption and pain reporting, i.e. that the lowest and highest categories of alcohol consumption had higher levels of pain reporting than those in the 'moderate' category of alcohol consumption.

\section{Methods}

The UK Biobank is a large cohort study which recruited more than 500,000 people between the ages of 40 and 69 registered with a National Health Service general practitioner in the UK. Participants attended assessment centres in 22 cities in England, Scotland and Wales where they completed touchscreen questionnaires on health and lifestyle. The activities under UK Biobank are governed by an Ethics and Governance Framework, overseen by an Ethics and Governance Council (www.egcukbiobank.org.uk). The core scientific protocol and operational procedures of the UK Biobank resource, as well as proposed used of it, have approval from appropriate ethics committees.

Pain

Participants were asked if they had any pain in the last month in regional body sites, or to indicate if they had pain all over the body. For each site indicated they were asked whether pain had last for 3 months or more. For the current analysis, if a participant answered they had pain all over the body that had lasted for 3 months or more, they were classed as someone with CWP.

\section{Alcohol consumption}

Participants were first asked "-About how often do you drink alcohol?'”-. If a current non-drinker they were then asked whether they previously drank and if so, to select their reason for stopping from a list: 'Illness or ill health', 'Doctor's advice', 'Health precaution', 'Financial reasons', 'Other reason', 'Do not know', or 'Prefer not to answer'. For analysis the first three options were combined as 'lllness or Health Precaution', and the remainder as 'Other reasons'. Those who said they were currently drinking were asked if they currently drank more, less or the same compared to 10 years ago. Those who said they had reduced their drinking were asked their reason for reducing consumption from the same list given to those who had stopped drinking. The same classification of categories for those reducing consumption were used as those giving up drinking. Current drinkers were asked how much they drank per week, or per month if drinking less frequently than weekly, of white wine, red wine, beer or 
cider, spirits or liquers, and fortified wine, in appropriate measures, i.e., how many glasses or pints. Typical value in units were chosen for each alcohol-type and an overall consumption level was calculated for current drinkers. (For example, 1 glass of red wine was equal to $125 \mathrm{ml}$, and at $13 \%$ alcohol by volume was taken to be 1.625 units of alcohol). One was added to the number of units consumed per week (so that non-drinkers would have a value of 1 , to give a natural $\log$ of 0 ) and natural logarithms were taken (so that a dose-response relationship between CWP and alcohol could be plotted). Categories of these value were calculated in increments of 0.5 . For the stratified analyses, categories were combined where total numbers in a category were low.

\section{Adjusting variables}

Factors were chosen which were known to be risk factors for pain and were likely to be associated with alcohol consumption. These were: age (years) [21]; Body Mass Index (BMI) [25]; education (having a university degree or not) [19]; deprivation (Townsend Index [24]) [17]; social networks (frequency of visits by family and friends) [3]; mood ('Do you ever feel miserable for no reason?', and 'Do you often feel fed-up?') [16]; loneliness ('Do you often feel lonely?') [4]; smoking (Never smoker/Tried once or twice/Previous occasional smoking/Previous smoking most days/Current occasional smoker/Current smoker most days) [20]; ethnicity (White/Mixed/Asian or Asian British/Black or Black British/Chinese/Other ethnic group) [16]; and employment status [16]. Adjustment was also made for assessment centre.

\section{Statistical Analysis}

As prevalence of CWP has consistently been shown to be more common in females, and because levels of exposure to alcohol differed between males and females, analyses were conducted separately by gender. The proportion of people reporting CWP was calculated in each category of drinking status (i.e. drinker/non-drinker and change compared to 10 years ago). Logistic regression was used to calculate ORs, and adjORs for CWP in each category compared to current drinkers with no change in consumption. As prevalence of CWP is low, odds ratios are a good approximation for risk ratios. The proportion of people reporting CWP was then calculated for each level of alcohol consumption (and logistic regression used to calculate ORs and 95\% Confidence Intervals (Cls)). The reference category in males was log units plus one between (males 3 and -3.5 (- - corresponding to 19.1-32.1 units/wk), and in females between 2 and $-2.5 \_$,-corresponding to 6.4-11.2 units/wk). These were chosen as they were the modal consumption categories. The analysis was then stratified by change in alcohol consumption since 10 years ago, and reasons for change in consumption. ORs were plotted on graphs at the midpoint of a consumption category to show the dose-response relationship [13]. A sensitivity analysis was carried out including those who had missing data on the adjusting variables. Among 
drinkers, weekly consumption of red wine, white wine, beer or cider, and spirits was categorised and ORs and adjORs of CWP were calculated and plotted.

\section{Results}

502,656 people completed the UK Biobank recruitment questionnaire. After removing those with missing data (i.e.-missing pain status $n=4,610$, missing adjusting variables $n=44,473$, drinking monthly but not asked alcohol consumption $n=64,807$, not providing alcohol consumption $n=13,489$ ) 375,277 participants were included in the analysis. The median age was 58 years (interquartile range (IQR) 50 to 63 ), $51.7 \%$ were female, and $1.2 \%$ reported having CWP. Of those included in the analysis, $83.3 \%$ said they drink at least once a week, $7.4 \%$ drank less frequently, $4.3 \%$ were current non-drinkers but had previously drank, and 5.0\% had never drank. Median weekly consumption among males and females drinking weekly was 20.4 units (IQR 11.8-34.2), and 10.7 units (IQR 6.5-17.7) respectively.

\section{CWP and changes in drinking habits}

Reporting of CWP was least in those currently drinking and reporting no change in drinking habits (males $0.5 \%$, females $0.8 \%$ ) (Table 1 ) and greatest in those currently not drinking but reporting having been drinkers before (males 3.7\%, females $4.9 \%$ ). The excess remained after adjusting for potential confounding factors - adjOR 2.59 (95\% Cl 2.17-3.09) in males and 2.77 (95\% Cl 2.41-3.19) in females.

\section{The dose-response relationship between alcohol and CWP in males}

Among all males, the proportion reporting CWP (Table 2) was greater in those not drinking (3.2\%) compared to those in the reference category (0.7\%) - adjOR 2.16 (95\% Cl 1.84-2.55). Proportions were at similar levels in all those drinking low to moderate amounts (Figure 1a). There was an increase in CWP in those drinking more than 53.6 units per week compared to the reference category, but this was non-significant after adjustment (1.2\% with CWP in those drinking over 53.6 units per week, adjOR 1.07 95\% Cl 0.88-1.29). A similar dose-response pattern - either U-shaped or reversed-J-shaped - was also seen in those drinking more, the same, or less due to illness or as a health precaution, as 10 years previously (Figures $\mathbf{1 b}$-d). Specifically in those drinking the same as 10 years ago odds of reporting CWP was significantly elevated in non-drinkers (adjOR 2.53, 95\% $\mathrm{Cl} 1.78-3.60$ ) and in those with the highest consumption (adjOR 1.52, 95\% $\mathrm{Cl}$ 1.05-2.20).

\section{The dose-response relationship between alcohol and CWP in females}

Among females there was a decrease in proportion reporting CWP (Table 3) with increasing categories of consumption from non-drinkers (4.0\%) through to the reference category of those drinking 6.4 to 11.2 units per week (0.9\%) - adjOR 2.30 (95\% Cl 2.02-2.63). Proportions of those reporting CWP were also similar for increasing levels of consumption above the reference category (Figure 2a). This same 
pattern was seen in those drinking the same amount as 10 years previously (non-drinkers vs reference category adjOR 2.11, 95\% $\mathrm{Cl}$ 1.67-2.66) (Figure 2c) and those who had changed their consumption (Figures $\mathbf{2 b}, \mathbf{2 d}, \mathbf{2 e}$ ). Sensitivity analysis looking at the dose-response relationship of CWP with alcohol consumption including those participants for whom full adjusting data was not available did not alter the results (Table 6).

The dose-response relationship between consumption of different alcohol types and CWP among drinkers

In both male and female drinkers the strongest associations were with red wine consumption. There were increased odds of reporting CWP (Tables 4 and 5) in those not drinking red wine compared to those drinking 11.2-19.1 units per week (males: $1.3 \%$ vs $0.4 \%$, adjOR $1.7495 \% \mathrm{Cl} 1.28-2.35$; females: $1.3 \%$ v. $0.6 \%$ adjOR $1.4495 \% \mathrm{Cl} 1.12-1.85)$. There were increases in CWP with increasing amounts of beer/cider or spirits consumed but these were not significant after adjustment.

\section{Discussion}

This very large population-based study found that non-drinkers are more likely to report CWP than those drinking moderate amounts of alcohol. In males, the classic U- or J-shaped relationship was found with increased reporting of CWP in the very lowest and highest categories of consumption. In females there were reduced odds of reporting CWP across increasing categories of consumption. Furthermore similar dose-response patterns were shown when looking at sub-groups of people based on changes in drinking habits, specifically those who had the same consumption as 10 years previously, which might suggest that the observed pattern is not due to people changing their drinking habits due to a change in pain/health status. Examination of specific sources of alcohol showed that the dose-response relationship was mainly observed for red wine.

There are a number of limitations of cross-sectional observational surveys with self-report measures. Firstly, there may be some reporting bias and respondents may underestimate the amount of alcohol they drink, perhaps for reasons of social desirability. However, the reported levels of alcohol consumption were in the normal range. Before exclusions for missing data the proportion of males drinking over 21 units per week was 37\%, and over 50 units was $9 \%$. The proportions given for these categories in males between 45 and 64 in the General Lifestyle Survey (GLF) of 2010 [5] were 30\% and 9\%. In females in the UK Biobank, the proportion drinking over 14 units a week was $22 \%$ and the proportion over 35 units a week was $3 \%$, in comparison to $20 \%$ and $4 \%$ in the GLF. The design of this study also relies on the accurate reporting of change in drinking status from 10 years previously, although interestingly the results observed in this group were similar to all subjects included. A selection bias may have also be an issue given that an important proportion of participants were 
excluded for incomplete data. This is only a problem if the association is only found in those who provide full data, but similar results were found in the sensitivity analysis which included those with missing adjusting variables. In the statistical analysis a large number of adjusting variables were used. These were used for adjustment as they are presumed confounders. However, it is possible that some of these third variables may actually be mediators of the observed effect in which case they should not have been used in adjustment and the observed relationship will have been underestimated [1]. There are more general problems with drawing causal inferences from observational studies, and observationalindeed findings often fail to replicate in randomised controlled trials (RCTs) [14]. A trial of alcohol use for chronic pain-however, would not be ethically or methodologically feasible. A study design which can be used when RCTs are impracticable is Mendelian randomisation [28]. For example, , a-Mendelian randomisation was used in a study on the effect of alcohol on cardiovascular disease in which the the findings of observational studies were reversed [8]. Mendelian randomisation studies could be used to assess the relationship between alcohol and chronic pain using variants of genes for enzymes involved in the metabolism of alcohol, and this approach seems the most promising to understand if the relationship is causal or not.

The results of this study confirm and build on those of previous studies which have found that painreporting is higher in non-drinkers than in drinkers. As a much larger study with similar prevalance estimates as other cohorts [16] this allows for more precise estimates of effect. It also provides information on a much greater number of potential confounders. One purpose of the study was to look at criteria that might help to support or oppose the causal relationship between alcohol and reduced risk of pain, particularly the biological gradient. A linear monotonic dose-response relationship was found in females but not in males although it was not strong. The gradient in females was not greatly different in drinkers who had reduced their drinking because of illness and those who had not changed their drinking. This suggests the observed relationship is not explained by people reducing consumption due to pain. This is the first time the relationship has been examined by type of alcohol consumed and the relationship found when looking at total consumption was only clearly observed with red wine. This result goes against a general effect of alcohol and suggests that some unmeasured factors might confound the observed relationship between red wine drinking and pain. In our sample, red wine drinkers were different to those drinking other types of alcohol. For example, in males drinking between 19.1 and 32.1 units per week, those who drank any red wine were more likely to have a university degree than those that did not drink red wine at all ( $44 \%$ vs $18 \%$ ), less likely to be unemployed ( $1 \%$ vs $3 \%$ ), and less likely to report smoking most days ( $5 \%$ vs $13 \%$ ).

If there were a general effect of alcohol on chronic pain then mechanisms would have to be considered. It has been suggested that the acute effects of alcohol on pain are through its action on 
gamma-Aminobutyric acid (GABA) neurons in the central nervous system. GABA is an inhibitory neurotransmitter that has a role in the mediation of pain and has also been found to have altered concentrations in the insular cortex of fibromyalgia patients. Other mechanisms have been proposed for the action of alcoholon pain that do not involve directly its effecton neurotransmission but instead its psychosocial effects which are known risk markers for chronic pain, including reduction of fearavoidance mechanisms, social integration, and stress-relief. If it was established that alcohol consumption was effective in preventing chronic pain, prospective studies would be required to determine whether mediators of the effect were neurobiological or psychosocial.

There is a strong, clear association between drinking alcohol and reduced likelihood of reporting pain. There is however still no convincing evidence that drinking alcohol causes people to be less likely to have pain. This study provides evidence that the- association is not explained by people in poor health reducing their alcohol consumption, and that the association is most clear for red wine consumption. Similar relationships of low to moderate alcohol consumption are seen with a wide range of outcomes [6] and avoidance of alcohol might be considered a (non-causative) risk marker for poor general health, of which pain is one feature. If however, alcohol consumption does affect pain, or if people who are at risk of having chronic pain are found to process alcohol differently resulting in them reducing their consumption, then these findings could be used to inform new as-targets

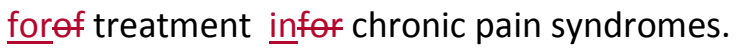

\section{Acknowledgements}

This research has been conducted using the UK Biobank resource, and was funded by the University of Aberdeen. The authors have no conflicts of interest to declare.

\section{References}

\section{References}

[1] Christenfeld NJ, Sloan RP, Carroll D, Greenland S. Risk factors, confounding, and the illusion of statistical control. Psychosom Med 2004;66:868-875.

[2] Chung $M$, Wang $C$. Can alcohol consumption be an alternative treatment for fibromyalgia? Arthritis Res Ther 2013;15:126. 
[3] Docking RE, Beasley M, Steinerowski A, Jones EA, Farmer J, Macfarlane GJ, Jones GT. The those $\geq 55$ years. British journal of pain 2014:2049463714527438.

[4] Docking RE, Fleming J, Brayne C, Zhao J, Macfarlane GJ, Jones GT, Cambridge City over-75s Cohort Study collaboration. Epidemiology of back pain in older adults: prevalence and risk factors for back pain onset. Rheumatology (Oxford) 2011;50:1645-1653.

[5] Dunstan S. General lifestyle survey overview: a report on the 2010 general lifestyle survey. : Office for National Statistics, 2012.

[6] Fekjaer HO. Alcohol-a universal preventive agent? A critical analysis. Addiction 2013;108:20512057.

[7] Fillmore KM, Kerr WC, Stockwell T, Chikritzhs T, Bostrom A. Moderate alcohol use and reduced mortality risk: Systematic error in prospective studies. Addiction Research \& Theory 2006;14:101132.

[8] Holmes MV, Dale CE, Zuccolo L, Silverwood RJ, Guo Y, Ye Z, Prieto-Merino D, Dehghan A, Trompet S, Wong A. Association between alcohol and cardiovascular disease: Mendelian randomisation analysis based on individual participant data. BMJ 2014;349:g4164.

[9] Hunt IM, Silman AJ, Benjamin S, McBeth J, Macfarlane GJ. The prevalence and associated features of chronic widespread pain in the community using the 'Manchester' definition of chronic widespread pain. Rheumatology (Oxford) 1999;38:275-279.

[10] Jones GT, Atzeni F, Beasley M, Flüß E, Sarzi-Puttini P, Macfarlane GJ. The prevalence of fibromyalgia in the general population: a comparison of the American College of Rheumatology 1990, 2010, and modified 2010 classification criteria. Arthritis \& Rheumatology 2015;67:568-575. 
[11] Kim CH, Vincent A, Clauw DJ, Luedtke CA, Thompson JM, Schneekloth TD, Oh TH. Association between alcohol consumption and symptom severity and quality of life in patients with fibromyalgia. Arthritis Res Ther 2013;15:R42.

[12] Knott CS, Coombs N, Stamatakis E, Biddulph JP. All cause mortality and the case for age specific alcohol consumption guidelines: pooled analyses of up to 10 population based cohorts. BMJ 2015;350:h384.

[13] Lang JM, Rothman KJ, Cann Cl. That confounded P-value. Epidemiology 1998;9:7-8.

[14] Lawlor DA, Smith GD, Bruckdorfer KR, Kundu D, Ebrahim S. Those confounded vitamins: what can we learn from the differences between observational versus randomised trial evidence? The Lancet 2004;363:1724-1727.

[15] Macfarlane GJ, Beasley M. Alcohol Consumption in Relation to Risk and Severity of Chronic Widespread Pain: Results From a UK Population-Based Study. Arthritis Care \& Research 2015;67:1297-1303.

[16] Macfarlane GJ, Beasley M, Smith BH, Jones GT, Macfarlane TV. Can large surveys conducted on highly selected populations provide valid information on the epidemiology of common health conditions? An analysis of UK Biobank data on musculoskeletal pain. British Journal of Pain 2015;9:203-212.

[17] Macfarlane GJ, Norrie G, Atherton K, Power C, Jones GT. The influence of socioeconomic status on the reporting of regional and widespread musculoskeletal pain: results from the 1958 British Birth Cohort Study. Ann Rheum Dis 2009;68:1591-1595.

[18] Mansfield KE, Sim J, Jordan JL, Jordan KP. A systematic review and meta-analysis of the prevalence of chronic widespread pain in the general population. Pain 2016;157:55-64. 
[19] McBeth J, Jones K. Epidemiology of chronic musculoskeletal pain. Best practice \& research Clinical rheumatology 2007;21:403-425.

[20] McBeth J, Lacey RJ, Wilkie R. Predictors of New-Onset Widespread Pain in Older Adults: Results From a Population-Based Prospective Cohort Study in the UK. Arthritis \& Rheumatology 2014;66:757-767.

[21] McBeth J, Macfarlane GJ, Hunt IM, Silman AJ. Risk factors for persistent chronic widespread pain: a community-based study. Rheumatology (Oxford) 2001;40:95-101.

[22] Stockwell T. A reply to Roerecke \& Rehm: continuing questions about alcohol and health benefits. Addiction 2013;108:428-429.

[23] Stockwell T, Chikritzhs T. Commentary: another serious challenge to the hypothesis that moderate drinking is good for health? Int J Epidemiol 2013;42:1792-1794.

[24] Townsend P, Phillimore P, Beattie A. Health and deprivation: inequality and the North. London: Croon Helm, 1988.

[25] VanDenKerkhof EG, Macdonald HM, Jones GT, Power C, Macfarlane GJ. Diet, lifestyle and chronic widespread pain: results from the 1958 British Birth Cohort Study. Pain Research and Management 2011;16:87-92.

[26] Wolfe F, Clauw DJ, Fitzcharles M, Goldenberg DL, Katz RS, Mease P, Russell AS, Russell IJ, Winfield JB, Yunus MB. The American College of Rheumatology preliminary diagnostic criteria for fibromyalgia and measurement of symptom severity. Arthritis care \& research 2010;62:600-610.

[27] Wolfe F, Smythe HA, Yunus MB, Bennett RM, Bombardier C, Goldenberg DL, Tugwell P, Campbell SM, Abeles M, Clark P. The American College of Rheumatology 1990 criteria for the classification of fibromyalgia. Arthritis \& Rheumatism 1990;33:160-172. 
[28] Yeung SL, Schooling CM. More ways to distinguish real from artefactual associations in observational studies. Int J Epidemiol 2014;43:1665-1666.

\section{Figures and Tables}

Table 1 Chronic Widespread Pain by changes in alcohol consumption

Table 2 Chronic Widespread Pain in males by alcohol consumption

Table 3 Chronic Widespread Pain in females by alcohol consumption

Table 4 Chronic Widespread Pain by consumption of different alcohol types in male drinkers

Table 5 Chronic Widespread Pain by consumption of different alcohol types in female drinkers

Table 6 Sensivity analysis, Chronic Widespread Pain by alcohol consumption including those with missing adjusting variables

Figure 1 Odds ratios ${ }^{2}$ for Chronic Widespread Pain by alcohol consumption category ${ }^{3}$ in males

Figure 2 Odds ratios ${ }^{2}$ for Chronic Widespread Pain by alcohol consumption category ${ }^{3}$ in females 
1

2

3

4

5

6

7

8

9

10

11

12

13

14

15

16

17

18

19

20

21

22

23

24

25

26

27

28

29

30

31

32

33

34

35

36

37

38

39

40

41

42

43

44

45

46

47

48

49

50

51

52

53

54

55

56

57

58

59

60

61

62

63

64

65

Alcohol consumption was associated with lowered reporting of chronic widespread pain in a large biobank. The association remained when looking at those without changed consumption. 




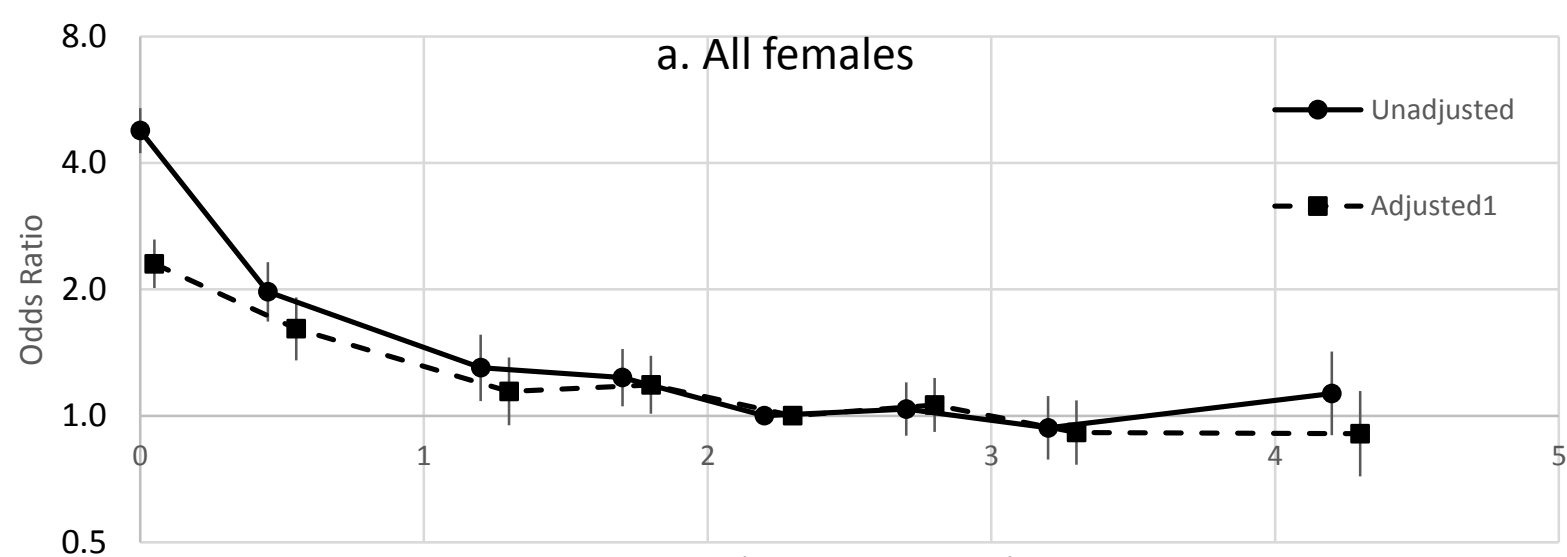

Log (Units per week + 1)
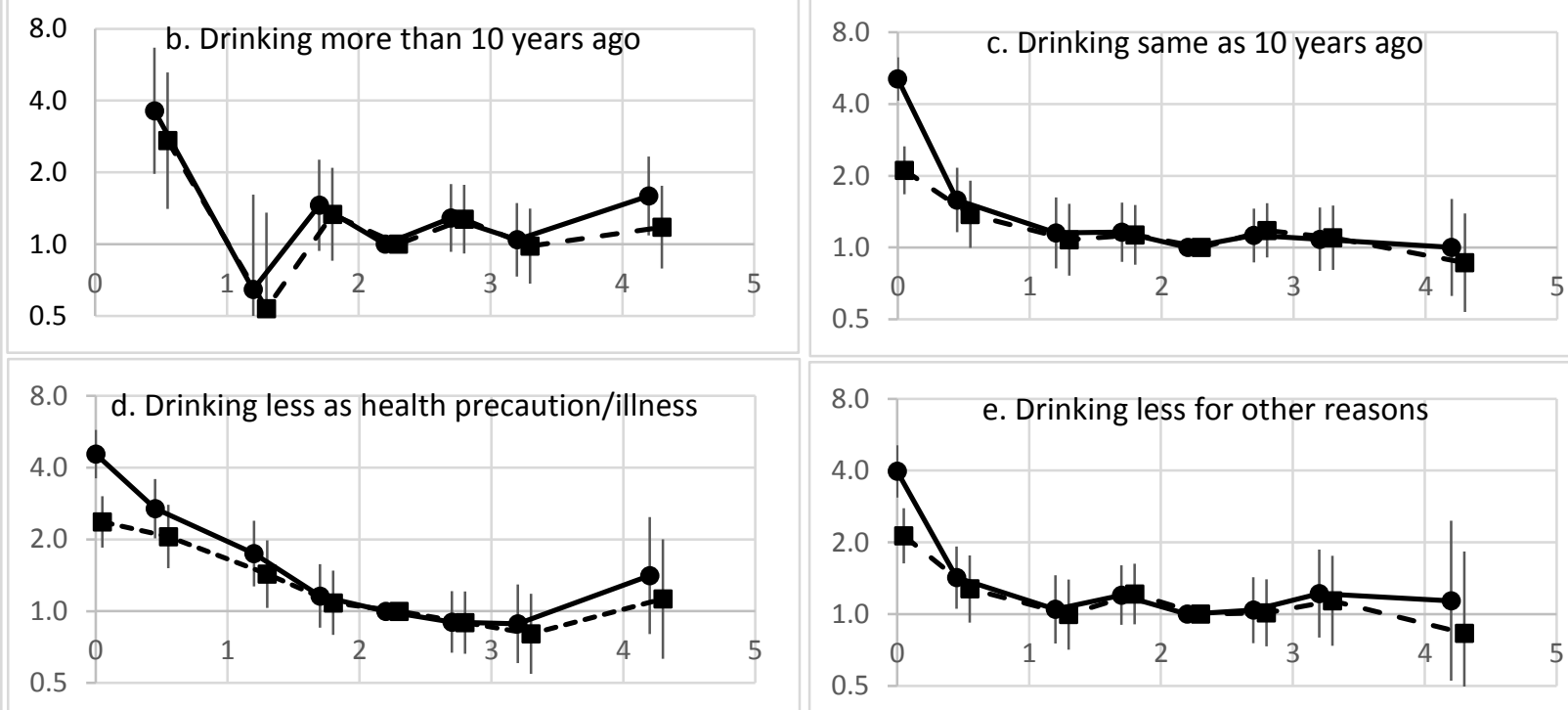
Table 1 CWP by changes in alcohol consumption

Males, $n=181,112$

\begin{tabular}{|c|c|c|c|c|c|}
\hline & Drinking status & Total & Number with CWP (\%) & OR & Adj OR \\
\hline \multirow{3}{*}{ Current } & No change & 63599 & $330(0.5)$ & 1 [Ref] & 1 [Ref] \\
\hline & & & & & \\
\hline & Drinking more than 10 years ago & 25197 & $167(0.7)$ & $1.28(1.06-1.54)$ & $1.09(0.90-1.32)$ \\
\hline \multirow[t]{2}{*}{ Drinkers } & & & & & \\
\hline & Drinking less than 10 years ago & 80088 & $807(1.0)$ & $1.95(1.72-2.22)$ & $1.33(1.17-1.52)$ \\
\hline Current non- & No change & 5137 & $123(2.4)$ & $4.70(3.82-5.80)$ & $2.36(1.87-2.98)$ \\
\hline drinkers & Previous drinker & 7091 & $263(3.7)$ & $7.38(6.27-8.70)$ & $2.59(2.17-3.09)$ \\
\hline
\end{tabular}

Females, $n=194,165$

\begin{tabular}{|c|c|c|c|c|c|}
\hline & Drinking status & Total & Number with CWP (\%) & OR & Adj OR \\
\hline \multirow{3}{*}{ Current } & No change & 65284 & $510(0.8)$ & 1 [Ref] & 1 [Ref] \\
\hline & & & & & \\
\hline & Drinking more than 10 years ago & 42108 & $326(0.8)$ & $0.99(0.86-1.14)$ & $0.92(0.80-1.06)$ \\
\hline \multirow{2}{*}{ Drinkers } & & & & & \\
\hline & Drinking less than 10 years ago & 64295 & $884(1.4)$ & $1.77(1.59-1.98)$ & $1.37(1.23-1.54)$ \\
\hline Current non- & No change & 13521 & $463(3.4)$ & $4.50(3.97-5.11)$ & $2.06(1.79-2.38)$ \\
\hline drinkers & Previous drinker & 8957 & $442(4.9)$ & $6.59(5.79-7.50)$ & $2.77(2.41-3.19)$ \\
\hline
\end{tabular}

Note: AdJOR is adjusted for age, BMI, education, deprivation, social networks, mood, loneliness, smoking, ethnicity, employment status, and assessment centre 
Table $\mathbf{2}$ CWP in males by alcohol consumption

\begin{tabular}{|c|c|c|c|c|c|}
\hline & Current weekly & & Number & & \\
\hline & consumption & Total & with CWP & OR (95\% Cl) & Adj OR (95\% Cl) \\
\hline & (units) & & (\%) & & \\
\hline & 0 & 12228 & $386(3.2)$ & $4.45(3.83-5.18)$ & $2.16(1.84-2.55)$ \\
\hline & $0-1.7$ & 4564 & $46(1.0)$ & $1.39(1.02-1.90)$ & $1.24(0.90-1.72)$ \\
\hline & $1.7-6.4$ & 17935 & $153(0.9)$ & $1.17(0.97-1.43)$ & $1.20(0.99-1.47)$ \\
\hline & $6.4-11.2$ & 23341 & $169(0.7)$ & $1.00(0.82-1.20)$ & $1.11(0.91-1.340$ \\
\hline$(n=181,112)$ & $11.2-19.1$ & 36639 & $241(0.7)$ & $0.90(0.76-1.07)$ & $0.98(0.82-1.16)$ \\
\hline & $19.1-32.1$ & 42495 & $309(0.7)$ & 1 [Ref] & 1 [Ref] \\
\hline & $32.1-53.6$ & 29437 & $219(0.7)$ & $1.02(0.86-1.22)$ & $0.94(0.79-1.12)$ \\
\hline & More than 53.6 & 14473 & $167(1.2)$ & $1.59(1.32-1.93)$ & $1.07(0.88-1.29)$ \\
\hline & $0-1.7$ & 134 & $2(1.5)$ & $3.07(0.73-12.92)$ & $3.30(0.67-13.72)$ \\
\hline & $1.7-6.4$ & 947 & $11(1.2)$ & $2.38(1.20-4.72)$ & $2.50(1 . .23-5.10)$ \\
\hline Drinking & $6.4-11.2$ & 2047 & $10(0.5)$ & $1.00(0.49-2.02)$ & $1.09(0.53-2.23)$ \\
\hline more & $11.2-19.1$ & 4384 & $23(0.5)$ & $1.07(0.63-1.82)$ & $1.17(0.68-2.01)$ \\
\hline$(n=25,197)$ & 19.1-32.1 & 6930 & $34(0.5)$ & 1 [Ref] & 1 [Ref] \\
\hline & $32.1-53.6$ & 6547 & $36(0.5)$ & $1.12(0.70-1.79)$ & $1.00(0.62-1.62)$ \\
\hline & More than 53.6 & 4208 & $51(1.2)$ & $2.49(1.61-3.85)$ & $1.30(0.82-2.07)$ \\
\hline
\end{tabular}

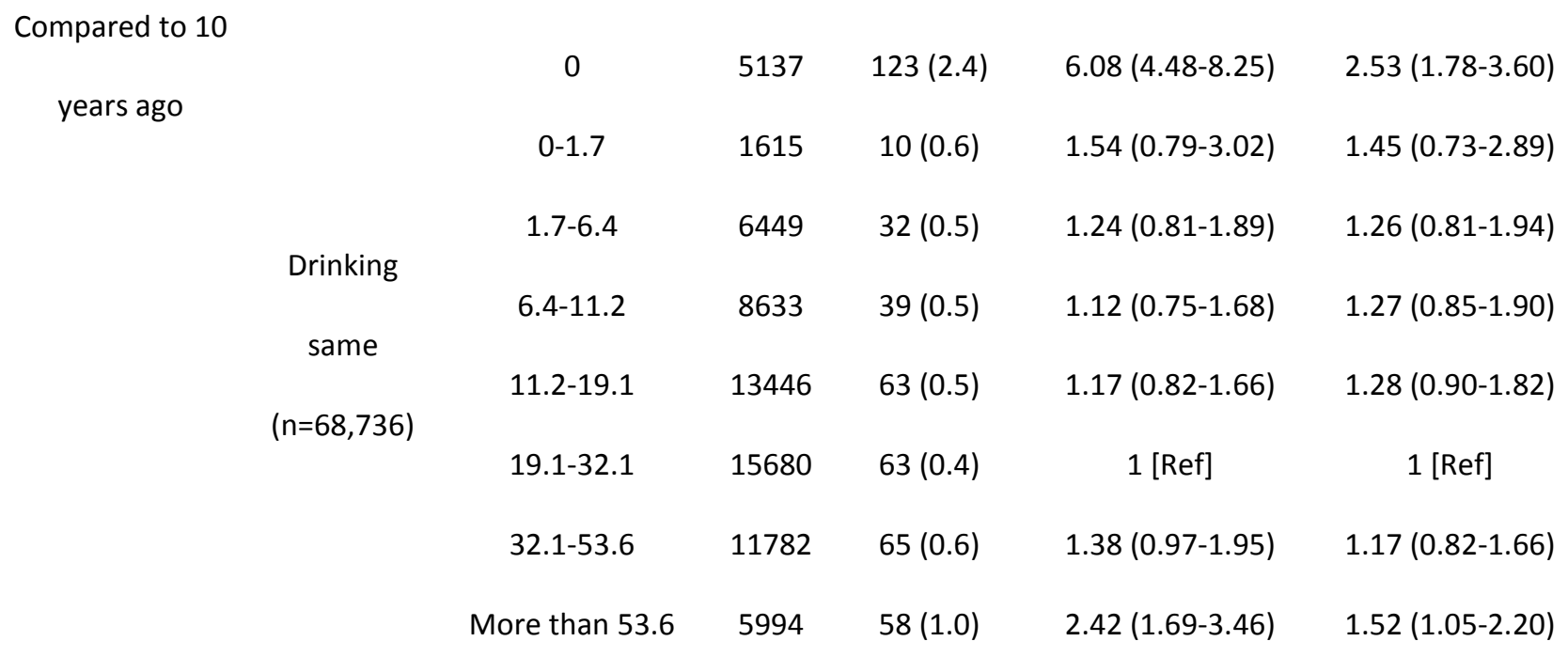




\begin{tabular}{|c|c|c|c|c|c|}
\hline & 0 & 3857 & $175(4.5)$ & $3.71(2.93-4.17)$ & $1.97(1.53-2.53)$ \\
\hline \multicolumn{6}{|l|}{ Drinking } \\
\hline \multirow{2}{*}{\multicolumn{6}{|c|}{$1.99(1.28-3.11)$}} \\
\hline & & & & & \\
\hline \multicolumn{6}{|r|}{1.44 (1.05-1.98) } \\
\hline \multirow{2}{*}{\multicolumn{6}{|c|}{$0.98(0.71-1.36)$}} \\
\hline & & & & & \\
\hline \multicolumn{6}{|l|}{ as health } \\
\hline \multirow{2}{*}{\multicolumn{6}{|c|}{ precaution }} \\
\hline & & & & & \\
\hline \multirow{3}{*}{$(n=37,087)$} & $32.1-53.6$ & 5439 & $67(1.2)$ & $0.97(0.72-1.32)$ & $0.90(0.66-1.22)$ \\
\hline & More than 53.6 & 2108 & 39 (1.9) & $1.47(1.02-2.12)$ & $1.0390 .71-1.50)$ \\
\hline & 0 & 3234 & $88(2.7)$ & $3.09(2.31-4.14)$ & $1.98(1.45-2.70)$ \\
\hline Drinking & $0-1.7$ & 1851 & $10(0.5)$ & $0.60(0.31-1.15)$ & $0.63(0.32-1.23)$ \\
\hline less for & $1.7-6.4$ & 7042 & $44(0.6)$ & $0.70(0.49-0.99)$ & $0.82(0.57-1.18)$ \\
\hline other & $6.4-11.2$ & 8160 & $64(0.8)$ & $0.87(0.64-1.20)$ & $1.09(0.79-1.51)$ \\
\hline reasons/ & $11.2-19.1$ & 11261 & $59(0.5)$ & $0.58(0.42-0.81)$ & $0.66(0.48-0.92)$ \\
\hline not known & 19.1-32.1 & 10712 & $96(0.9)$ & 1 [Ref] & 1 [Ref] \\
\hline \multirow[t]{2}{*}{$(n=50,092)$} & $32.1-53.6$ & 5669 & $51(0.9)$ & $1.00(0.71-1.410$ & $0.92(0.65-1.29)$ \\
\hline & More than 53.6 & 2163 & $19(0.9)$ & $0.98(0.60-1.61)$ & $0.69(0.42-1.15)$ \\
\hline
\end{tabular}

Note: AdJOR is adjusted for age, BMI, education, deprivation, social networks, mood, loneliness, smoking, ethnicity, employment status, and assessment centre. The log likelihood chi-square from model for all males including all covariates was 2761.56 with 45 degrees of freedom and $\mathrm{p}<0.0001$ and pseudo R-square was 0.1441). 
Table 3 CWP in females by alcohol consumption

\begin{tabular}{|c|c|c|c|c|c|}
\hline & Current weekly & & Number & & \\
\hline & consumption & Total & with CWP & OR $(95 \% \mathrm{Cl})$ & Adj OR (95\% Cl) \\
\hline & (units) & & (\%) & & \\
\hline & 0 & 22478 & $905(4.0)$ & $4.78(4.22-5.40)$ & $2.30(2.02-2.63)$ \\
\hline & $0-1.7$ & 14611 & $249(1.7)$ & $1.97(1.68-2.32)$ & $1.61(1.36-1.91)$ \\
\hline & $1.7-3.5$ & 15397 & $174(1.1)$ & $1.30(1.08-1.56)$ & $1.14(0.95-1.38)$ \\
\hline & $3.5-6.4$ & 26130 & $280(1.1)$ & $1.23(1.05-1.44)$ & 1.19 (1.01-1.39) \\
\hline All $(n=194,165)$ & & & & & \\
\hline & $6.4-11.2$ & 41227 & $359(0.9)$ & 1 [Ref] & $1[$ Ref $\}$ \\
\hline & $11.1-19.1$ & 40490 & $366(0.9)$ & $1.04(0.90-1.20)$ & $1.06(0.92-1.23)$ \\
\hline & $19.1-32.1$ & 24382 & $199(0.8)$ & $0.94(0.79-1.11)$ & $0.91(0.77-1.09)$ \\
\hline & More than 32.1 & 9450 & $93(1.0)$ & $1.13(0.90-1.42)$ & $0.91(0.72-1.15)$ \\
\hline & $0-1.7$ & 578 & $13(2.2)$ & $3.63(1.97-6.67)$ & $2.72(1.41-5.25)$ \\
\hline & $1.7-3.5$ & 1227 & $5(0.4)$ & $0.65(0.26-1.61)$ & $0.54(0.21-1.36)$ \\
\hline & $3.5-6.4$ & 3388 & $31(0.9)$ & $1.46(0.94-2.26)$ & $1.34(0.85-2.09)$ \\
\hline Drinking more & & & & & \\
\hline$(n=42,108)$ & $6.4-11.2$ & 8886 & $56(0.6)$ & $1[$ Ref $\}$ & 1 [Ref] \\
\hline & 11.1-19.1 & 12585 & $102(0.8)$ & $1.29(0.93-1.79)$ & $1.27(0.91-1.77)$ \\
\hline & $19.1-32.1$ & 10346 & $68(0.7)$ & $1.04(0.73-1.49)$ & $0.98(0.68-1.41)$ \\
\hline & More than 32.1 & 5098 & $51(1.0)$ & 1.59 (1.09-2.33) & $1.18(0.79-1.76)$ \\
\hline Compared to & 0 & 13521 & $463(3.4)$ & $5.09(4.13-6.28)$ & $2.11(1.67-2.66)$ \\
\hline 10 years ago & $0-1.7$ & 5779 & $63(1.1)$ & $1.58(1.16-2.16)$ & $1.38(1.00-1.91)$ \\
\hline Drinking same & $1.7-3.5$ & 5910 & $47(0.8)$ & $1.15(0.82-1.62)$ & $1.08(0.76-1.53)$ \\
\hline as 10 years & $3.5-6.4$ & 10368 & $83(0.8)$ & $1.16(0.87-1.54)$ & $1.13(0.85-1.51)$ \\
\hline ago & $6.4-11.2$ & 15910 & $110(0.7)$ & 1 [Ref] & $1[$ Ref $\}$ \\
\hline$(n=78,805)$ & 11.1-19.1 & 15467 & $120(0.8)$ & $1.12(0.87-1.46)$ & $1.18(0.91-1.53)$ \\
\hline & 19.1-32.1 & 8815 & $66(0.7)$ & $1.08(0.80-1.47)$ & $1.10(0.81-1.50)$ \\
\hline & More than 32.1 & 3035 & $21(0.7)$ & $1.00(0.63-1.60)$ & $0.86(0.54-1.39)$ \\
\hline & 0 & 4103 & $261(6.4)$ & $4.55(3.60--5.76)$ & $2.37(1.85-3.03)$ \\
\hline
\end{tabular}




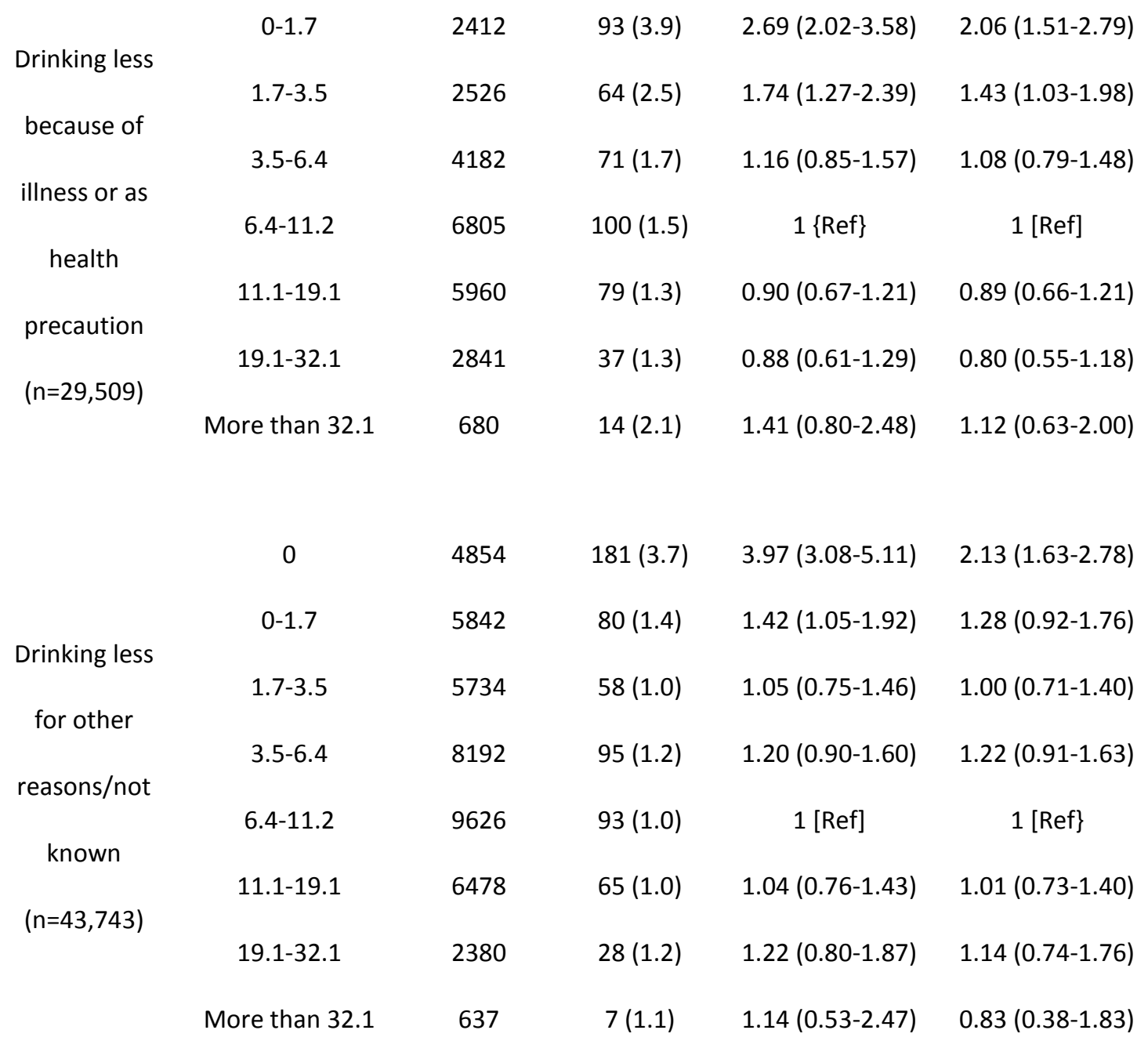

Note: AdJOR is adjusted for age, BMI, education, deprivation, social networks, mood, loneliness, smoking, ethnicity, employment status, and assessment centre. The log likelihood chi-square from model for all females including all covariates was 3800.34 with 45 degrees of freedom and $p<0.0001$ and pseudo R-square was 0.1367 . 
Table 4 CWP by consumption of different alcohol types in male drinkers $(n=168,884)$

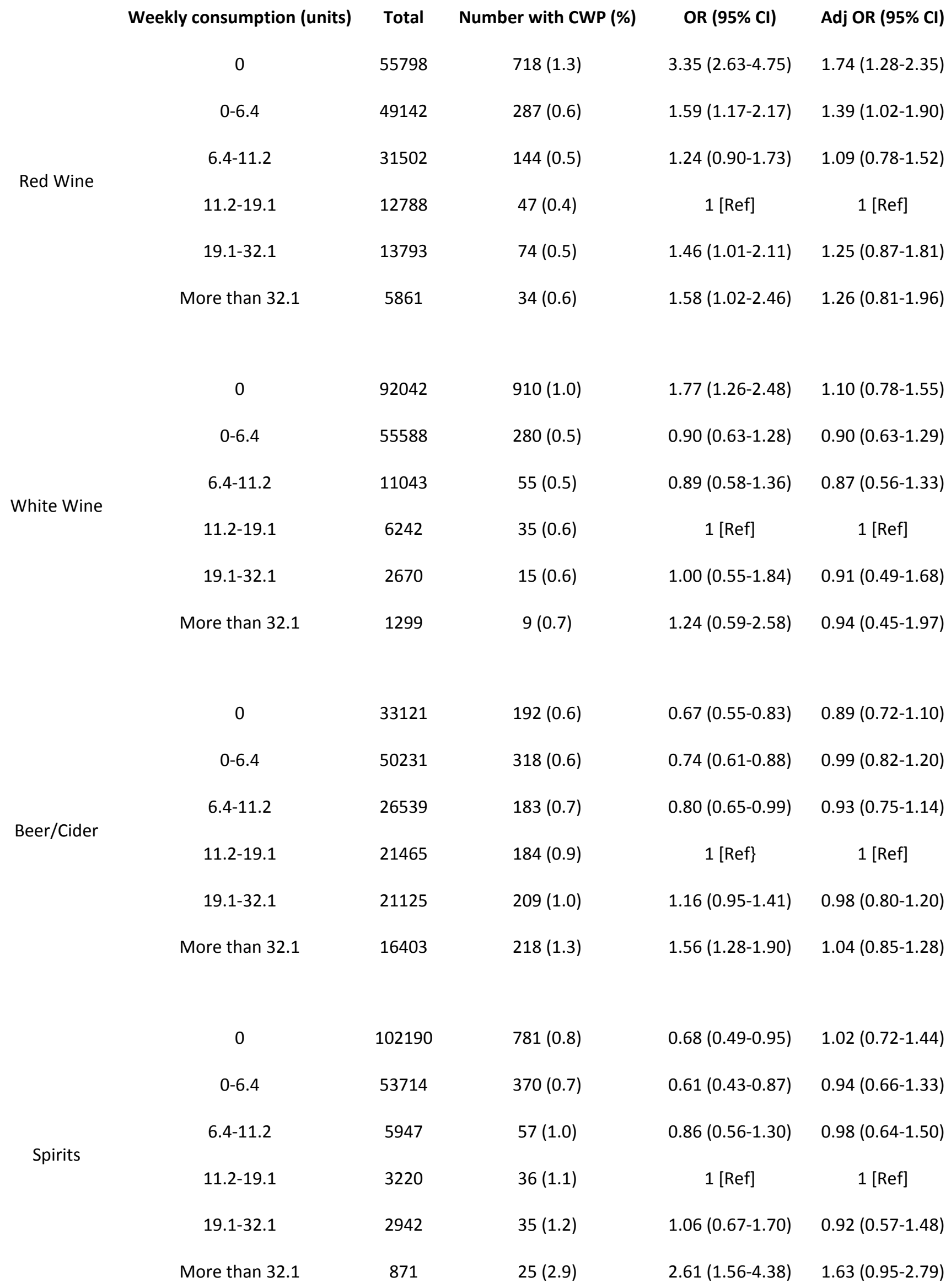

Note: AdJOR is adjusted for age, BMI, education, deprivation, social networks, mood, loneliness, smoking, ethnicity, employment status, and assessment centre 
Table 5 CWP by consumption of different alcohol types in female drinkers $(n=171,687)$

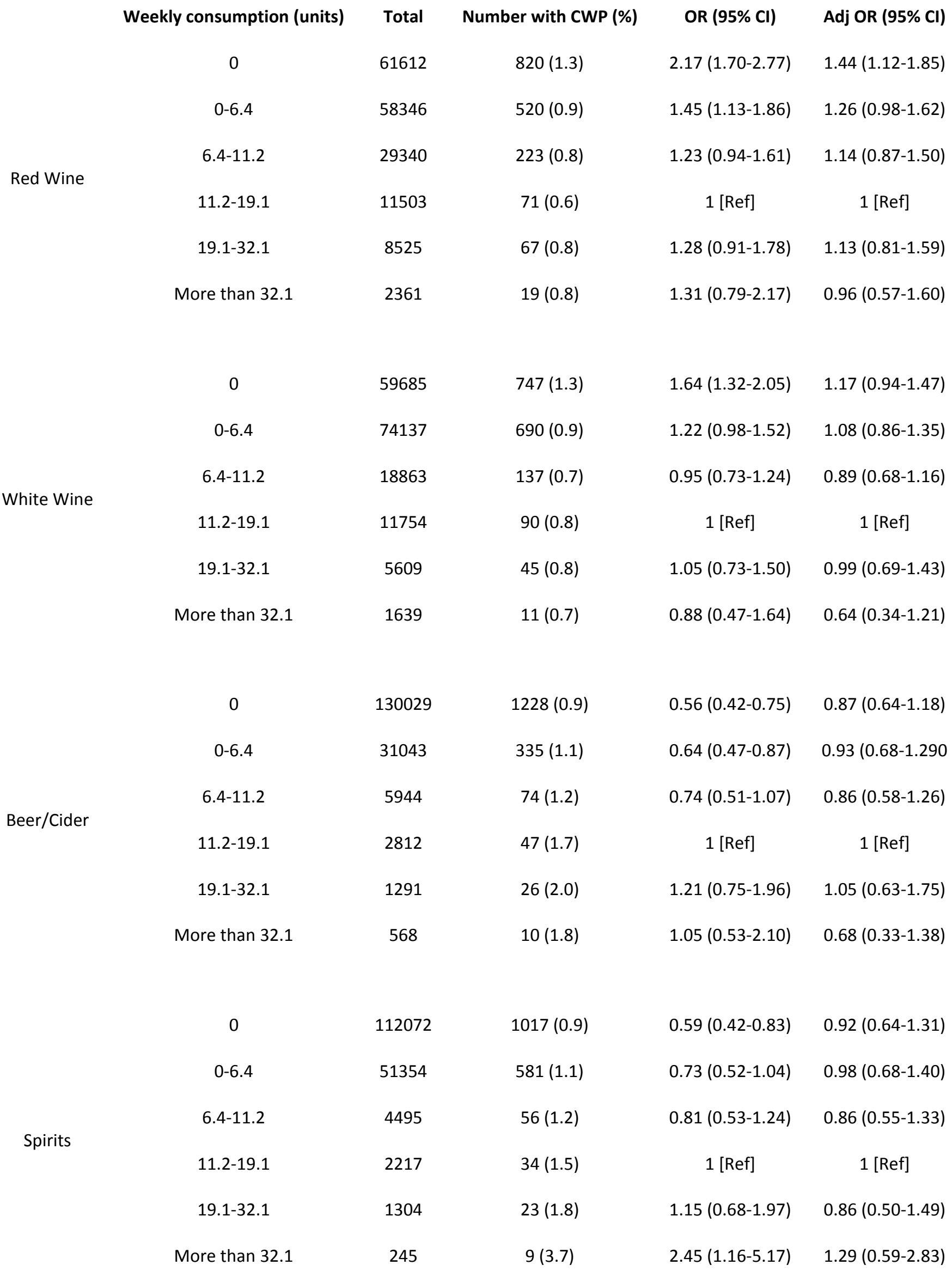

Note: AdJOR is adjusted for age, BMI, education, deprivation, social networks, mood, loneliness, smoking, ethnicity, employment status, and assessment centre 
Table 6 Sensitivity analysis, CWP by alcohol consumption including those with missing adjusting variables

Males $(n=196,529)$

$\begin{array}{cccc}\text { Weekly consumption (units) } & \text { Total } & \text { Number with CWP (\%) } & \text { OR (95\% Cl) } \\ 0 & 14346 & 500(3.5) & 4.69(4.09-5.39) \\ 0-1.7 & 5036 & 60(1.2) & 1.57(1.19-2.06) \\ 1.7-6.4 & 19539 & 177(0.9) & 1.19(0.99-1.42) \\ 6.4-11.2 & 25145 & 199(0.8) & 1.04(0.87-1.23) \\ 11.2-19.1 & 39426 & 273(0.7) & 0.91(0.77-1.06) \\ 19.1-32.1 & 45711 & 349(0.8) & 1[\text { Ref }] \\ 32.1-53.6 & 31617 & 241(0.8) & 1.00(0.85-1.18) \\ \text { More than } 53.6 & 15709 & 188(1.2) & 1.57(1.32-1.88)\end{array}$

Females $(n=209,708)$

Weekly consumption (units)

Total

25651

15909

16618

28115

44175

43244

25939

10057
Number with CWP 9\%)

1104 (4.3)

282 (1.8)

$193(1.2)$

307 (1.1)

409 (0.9)

406 (0.9)

$211(0.8)$

101 (1.0)
OR $(95 \% \mathrm{Cl})$

4.81 (4.29-5.40)

$1.93(1.66-2.25)$

$1.26(1.06-1.49)$

$1.18(1.02-1.37)$

1 [Ref]

$1.01(0.88-1.16)$

$0.88(0.74-1.04)$

1.09 (0.87-1.35) 